\title{
Performance analysis of superconducting rf cavities for the CERN rare isotope accelerator
}

\author{
S. Calatroni, ${ }^{*}$ A. Miyazaki, G. Rosaz, A. Sublet, and W. Venturini Delsolaro \\ CERN, 1211 Geneva 23, Switzerland
}

\author{
R. Vaglio \\ Dipartimento di Fisica, Università di Napoli Federico II, CNR-SPIN and INFN, 80125 Napoli (NA), Italy

\section{Palmieri} \\ Legnaro National Laboratories-Istituto Nazionale Fisica Nucleare (INFN), 35020 Legnaro (PD), Italy
} (Received 14 June 2016; published 23 September 2016)

\begin{abstract}
The first cryomodule of the new HIE-ISOLDE rare isotope accelerator has recently been commissioned with beam at CERN, with the second cryomodule ready for installation. Each cryomodule contains five superconducting low-beta quarter wave cavities, produced with the technology of sputtering a thin niobium film onto the copper substrate $(\mathrm{Nb} / \mathrm{Cu})$. This technology has several benefits compared to the bulk niobium solution, but also drawbacks among which the most relevant is the increase of surface resistance with accelerating field. Recent work has established the possible connection of this phenomenon to local defects in the $\mathrm{Nb} / \mathrm{Cu}$ interface, which may lead to increased thermal impedance and thus local thermal runaway. We have analyzed the performance of the HIE-ISOLDE cavities series production, as well as of a few prototypes', in terms of this model, and found a strong correlation between the rf properties and one of the model characteristic quantities, namely the total surface having increased interface thermal impedance.
\end{abstract}

DOI: 10.1103/PhysRevAccelBeams.19.092002

\section{INTRODUCTION}

The HIE-ISOLDE project [1] includes an energy upgrade of the ISOLDE radioactive ion facility at CERN by means of a superconducting booster LINAC based on Quarter Wave Resonators (QWR). For these superconducting cavities CERN has chosen the $\mathrm{Nb} / \mathrm{Cu}$ technology [2], already used in LEP, LHC and ALPI, see Fig. 1.

After a development phase $[3,4]$ the production of cavities started at CERN following established protocols [5]. Although the prototype cavities had reached performances well above the HIE ISOLDE specifications, the series cavities displayed systematically higher surface resistances. This loss of performance was correlated with the presence of defects on the copper surface of the cavities as manufactured [6]. Optical inspection often revealed microscopic cracks close to the heat affected zone of the electron beam weld, in the high magnetic field region of the quarter wave resonator. It has been conjectured that chemicals from the polishing steps might be trapped in these cracks and locally contaminate the niobium layer and increase its surface resistance. It has to be noted that, even

\footnotetext{
*Corresponding author. sergio.calatroni@cern.ch

Published by the American Physical Society under the terms of the Creative Commons Attribution 3.0 License. Further distribution of this work must maintain attribution to the author(s) and the published article's title, journal citation, and DOI.
}

for the prototype cavities, the BCS surface resistance only accounts for a minor fraction of the observed rf losses $(15 \%-30 \%)$. This is mainly due to the low $(101 \mathrm{MHz}) \mathrm{rf}$ frequency, despite $4.5 \mathrm{~K}$ operation. A first component of the residual surface resistance, which could be identified in our case, was related to magnetic flux trapped during the
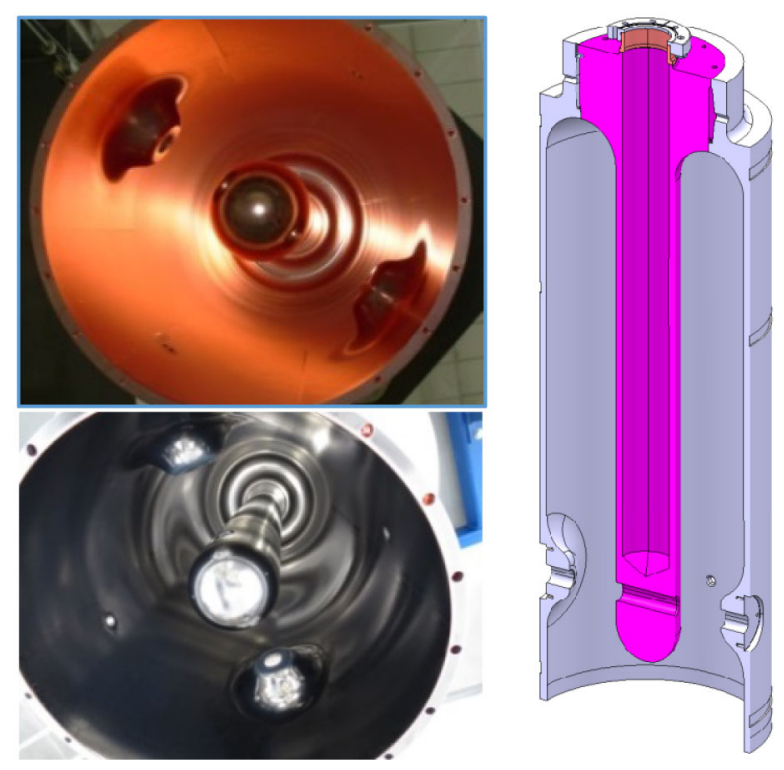

FIG. 1. The HIE-ISOLDE QWR cavities, before (top left) and after (bottom left) coating, and 3D illustration of the cavity geometry (right). 


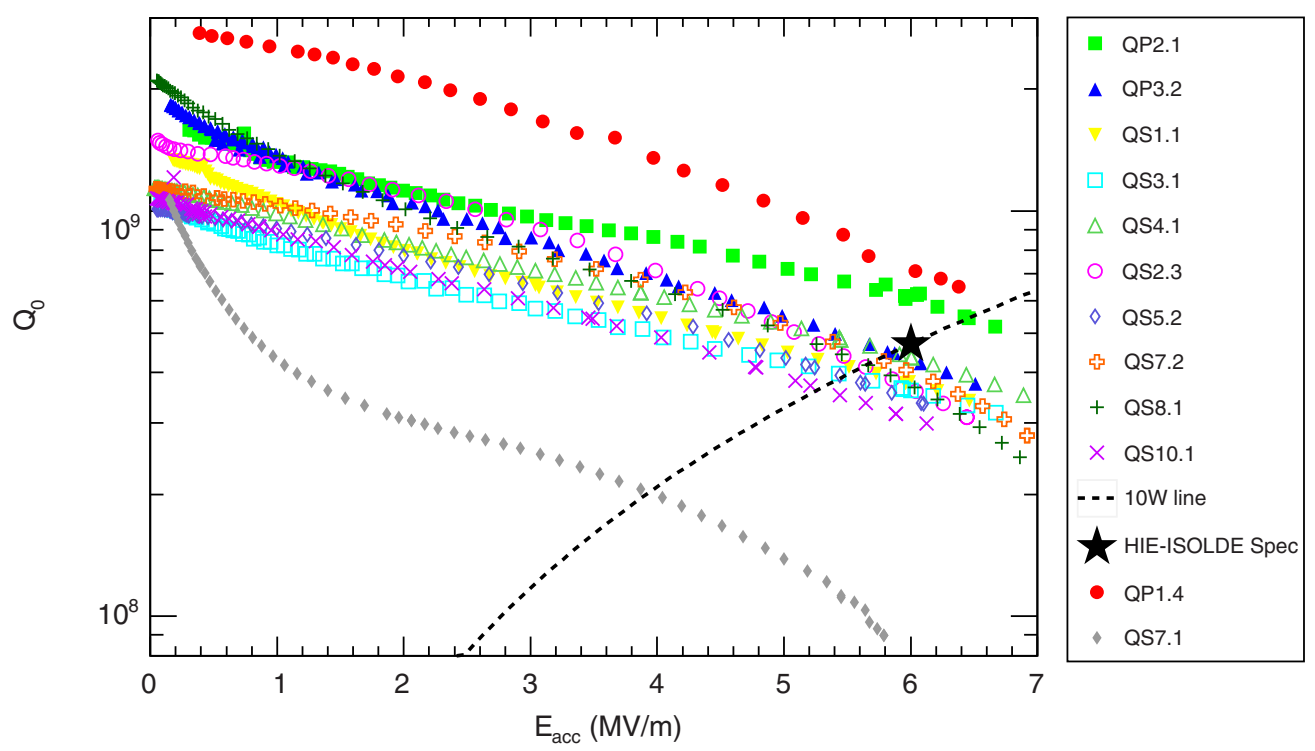

FIG. 2. $Q(E)$ data of all cavities of interest for this study, namely the 10 cavities installed in the first two cryomodules, the reference cavity QP1.4 and the test cavity QS7.1. Details of the nomenclature are explained in the text.

transition to the superconducting state. The main part of this flux does not come from the ambient magnetic field, but is linked to the lack of temperature homogeneity when the cavity is cooled through Tc. This was shown by cooling the same cavities with variable temperature gradients [6]. All cavity measurements reported here (Fig. 2) were done following a cooldown procedure whereby the thermal gradient across the cavity is minimized. However, even when extrapolating the measured data to zero temperature gradient, there remains a significant fraction of surface resistance of unknown origin, and this part was found to be correlated to the presence of observable surface defects.

The purpose of this paper is to apply to our data set a recently proposed model for the $Q$ slope of coated cavities in terms of locally increased thermal impedance at the film/ substrate interface [7], as this seems a promising candidate to explain the observed extra losses.

\section{COATING PROCESS}

The coating process is based on DC-bias diode sputtering using Ar as sputter gas at a pressure of $0.2 \mathrm{mbar}$ and at high temperature (from $300^{\circ} \mathrm{C}$ up to $620^{\circ} \mathrm{C}$ ), using a cylindrical $\mathrm{Nb}$ cathode coaxial to the cavity and powered at a nominal power of $8 \mathrm{~kW}$ [4]. Due to the high power and high temperature process the coating is done in 14 successive runs of about $25 \mathrm{~min}$ each, separated by a cool down of about $5 \mathrm{~h} 35 \mathrm{~min}$, resulting in a net coating time of about $6 \mathrm{~h}$ over 4 days. The $\mathrm{Nb}$ film thickness obtained presents a nonuniform profile over the cavity inner surface, ranging from an average of $2 \mu \mathrm{m}$ on the outer conductor to an average of $7 \mu \mathrm{m}$ on the inner conductor. It is assumed that a minimum thickness of $1.5 \mu \mathrm{m}$ is sufficient to guarantee the superconductive and rf properties of the film and shield the copper substrate, also taking into account all possible imperfections due to morphology, impurities, etc.. The thickness nonuniformity is mostly linked to the inner/ outer cavity geometry, the cathode/substrate aspect ratio and the inner/outer plasma discharge distribution.

The nomenclature chosen for the prototype and series cavities is QPX.Y and QSX.Y respectively, with X the cavity serial number and $\mathrm{Y}$ the $\mathrm{Nb}$ coating process number, as the $\mathrm{Nb}$ layer can be chemically stripped away and the cavity recoated several times [2]. The copper substrates were manufactured at CERN for the prototype "QP" cavities and by the industry for the series "QS" cavities [8]. Cavity QP1.4 represents our reference coating from the development phase, which led to the coating procedure described above. Over the two years production period, a total of 14 coatings have been made on 10 other substrates: 4 cavities have been coated twice due to bad initial rf performances (QP3, QS2, QS5 and QS7, not reported here with the exception of Q7.1). Typically a period of about two months is necessary [5], from the copper substrate reception at CERN, in order to obtain a coated cavity and verify its rf performance.

For most of the production the hardware coating setup (UHV chamber, Nb cathode, DC power supplies, etc.) and the process (production steps, coating recipe) have been kept identical, except for QP2 and QS10 substrates, which have seen an additional annealing prior to coating. Furthermore, two substrates, namely QS7.1 and QS8.1, have been used to investigate the effect of the bias potential on the film structure and rf performances of the cavities. QS7.1 was used for a test using $50 \%$ higher bias voltage as compared to the baseline recipe, from $-80 \mathrm{~V}$ to $-120 \mathrm{~V}$, to densify the $\mathrm{Nb}$ layer. A cathode enlarged at the top was also used to increase the 



FIG. 3. FIB-SEM cross section images of HIE-ISOLDE coating at the top of the cavity with $-80 \mathrm{~V}$ bias (left) and $-120 \mathrm{~V}$ bias + wide cathode (right) showing the densification of the layer.
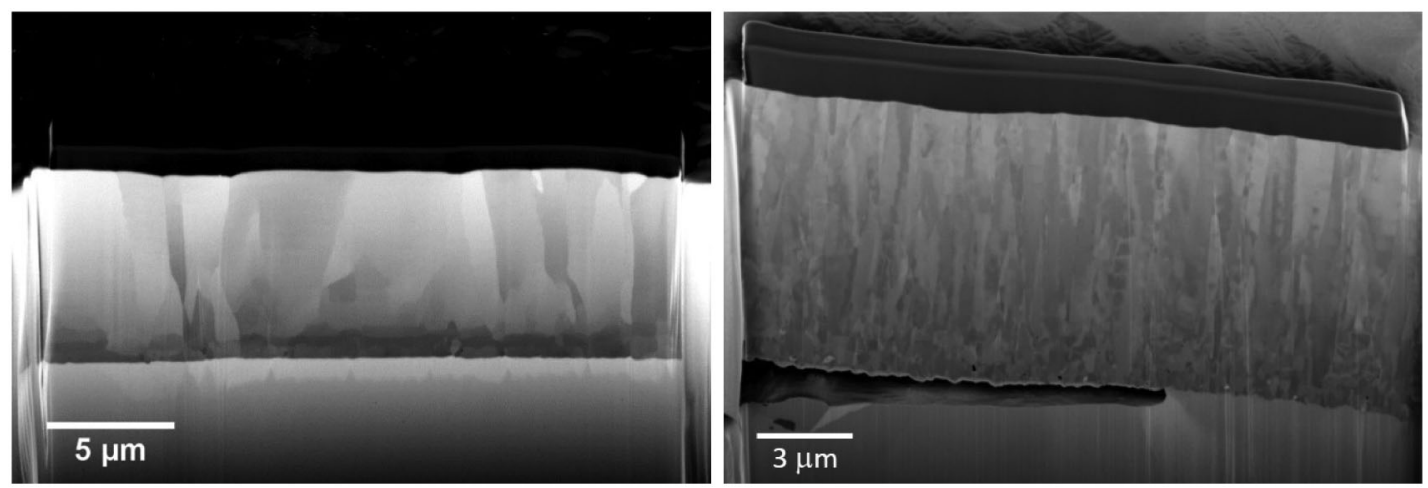

FIG. 4. FIB-SEM cross section images of HIE-ISOLDE coating at the middle of the antenna with $-80 \mathrm{~V}$ bias (left) and $-120 \mathrm{~V}$ bias (right) showing the delamination of the layer.

coating rate at the top of the cavity. The same configuration was used for cavity QS8.1 but with a bias of $-90 \mathrm{~V}$.

Increasing the substrate bias voltage by $50 \%$ from $-80 \mathrm{~V}$ to $-120 \mathrm{~V}$ and adjusting the cathode geometry facing the top of the cavity ( $4 \mathrm{~mm}$ wide instead of $2 \mathrm{~mm}$ ) improved consequently the density and thickness of the layer at this position, see Fig. 3. This happened however at the expense of the inner conductor part where the thin film analysis by FIB-SEM cross section showed delamination of the layer, see Fig. 4. Combination of thickness nonuniformity along the cavity and stress induced by the larger bias could explain this issue.

\section{THERMAL MODEL AND FITTING PROCEDURE}

The $Q$-slope problem strongly limits the performance of superconducting accelerating cavities, to a larger extent in thin film coated cavities [9]. In a recent paper it was shown that the $Q$-slope in thin films cavities can be related to enhanced thermal boundary resistance $R_{\mathrm{Nb} / \mathrm{Cu}}$ at the $\mathrm{Nb} / \mathrm{Cu}$ interface, due to poor thermal contact between the $\mathrm{Nb}$ film and the $\mathrm{Cu}$ substrate related to bad adhesion [7]. Assuming that thermal problems at the $\mathrm{Nb} / \mathrm{Cu}$ interface are the main source of the $Q$-slope, from the measured $Q$ vs rf field amplitude, through "inverse problem" techniques one can determine the distribution function $f\left(R_{\mathrm{Nb} / \mathrm{Cu}}\right)$ of thermal boundary resistances.

Summarizing, the presence of a large interface thermal resistance $R_{\mathrm{Nb} / \mathrm{Cu}}$ locally affects the value of the surface resistance $R_{s}$ so that we can write $R_{s}=R_{s}\left(T_{o}, E_{\mathrm{acc}}, R_{\mathrm{Nb} / \mathrm{Cu}}\right)$, where $T_{o}$ is the helium bath temperature and $E_{\text {acc }}$ is the rf accelerating field.

The measured cavity quality factor $Q$ will be related to the average value of $R_{s}$. The following relations hold:

$$
\begin{gathered}
Q=\frac{\Gamma}{\overline{R_{s}\left(T_{o}, E_{\mathrm{acc}}\right)}} \\
\overline{R_{S}\left(T_{o}, E_{\mathrm{acc}}\right)}=\int_{0}^{\infty} R_{s}\left(T_{o}, E_{\mathrm{acc}}, R_{\mathrm{Nb} / \mathrm{Cu}}\right) f\left(R_{\mathrm{Nb} / \mathrm{Cu}}\right) d R_{\mathrm{Nb} / \mathrm{Cu}} \\
\int_{0}^{\infty} f\left(R_{\mathrm{Nb} / \mathrm{Cu}}\right) d R_{\mathrm{Nb} / \mathrm{Cu}}=1
\end{gathered}
$$

Equation (1) allows the usual experimental determination of $\overline{R_{s}\left(T_{o}, E_{\text {acc }}\right)}$, with $\Gamma$ depending on cavity shape and determined by numerical codes. 
Equation (2) represents a classical first type Fredholm integral equation, and by well-known inverse problem methods can be "inverted" to extract $f\left(R_{\mathrm{Nb} / \mathrm{Cu}}\right)$ as was discussed in [7], knowing the function $R_{s}=R_{s}\left(T_{o}, E_{\mathrm{acc}}, R_{\mathrm{Nb} / \mathrm{Cu}}\right)$.

This last function can be easily calculated starting from the standard superconductor surface resistance expression:

$$
R_{S}(T)=\frac{A \omega^{2}}{T} \exp \left[-\frac{\Delta(T)}{k_{B} T}\right]+R_{o}
$$

where the temperature $T$ is the effective temperature at the cavity inner surface, which can be written as:

$$
\begin{aligned}
T & =T_{o}+\Delta T=T_{o}+R_{\mathrm{Nb} / \mathrm{Cu}} P_{r f} \\
& =T_{o}+R_{\mathrm{Nb} / \mathrm{Cu}} \frac{1}{2} R_{s}(T) H_{r f}^{2}
\end{aligned}
$$

$T_{o}$ is the helium bath temperature and $H_{r f}=B_{r f} / \mu_{o}=$ $k E_{\text {acc }} / \mu_{o}$, where $k$ represents the ratio between the maximum rf magnetic field at the inner cavity surface and the maximum accelerating field, and is a known constant depending on the specific cavity geometry (for simplicity we assume $R_{\mathrm{Nb} / \mathrm{Cu}}$ being temperature independent: including a realistic dependence would not change the results significantly). Finally we get:

$$
T=T_{o}+R_{\mathrm{Nb} / \mathrm{Cu}} \frac{1}{2} R_{s}(T)\left(\frac{k}{\mu_{o}}\right)^{2} E_{\mathrm{acc}}^{2}
$$

Equations (3)-(4) are nonlinear coupled equations and cannot be solved analytically. However, they can be solved by iteration. As discussed in [7] for each $R_{\mathrm{Nb} / \mathrm{Cu}}$ value the result shows an initial small parabolic increase of the surface resistance with the applied field, corresponding to a very small local surface temperature increase, followed at higher rf fields by a very sharp $R_{s}$ increase corresponding to a large surface temperature increase (thermal runaway process). When the surface temperature overcomes the superconducting critical temperature, we will have a "quench" of the superconducting state and the surface resistance will reach its normal state value $R_{n}$. The field at which the surface is

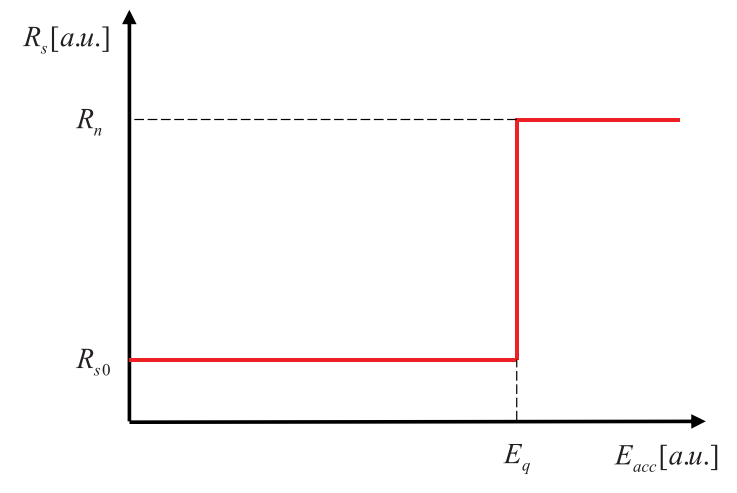

FIG. 5. Assumed step-like dependence for $R_{s}$ vs accelerating field $E_{\text {acc }}$.

driven in the normal state is defined here as the "quench field" $E_{q}$.

From inspection of Eq. (4) it is clear that the relation $R_{\mathrm{Nb} / \mathrm{Cu}} E_{q}^{2}=K$ always holds true.

The value of the constant $K$ depends on the superconducting cavity parameters and in particular on the intrinsic zero-field residual resistance, and can be evaluated by Eqs. (3)-(4).

We have observed that the solution of the inverse problem discussed above can be strongly simplified, keeping essentially the same level of accuracy, by assuming for the function $R_{s}=R_{s}\left(T_{o}, E_{\mathrm{acc}}, R_{\mathrm{Nb} / \mathrm{Cu}}\right)$ a step-like behavior as illustrated in Fig. 5:

$$
R_{s}= \begin{cases}R_{s 0} & E_{\mathrm{acc}} \leq E_{q} \\ R_{n} & E_{\mathrm{acc}}>E_{q}\end{cases}
$$

With $R_{s 0}=R_{s}\left(T_{o}, 0,0\right), R_{s 0} \ll R_{n}$ and the quench field $E_{q}$ given by:

$$
E_{q}=\sqrt{K / R_{\mathrm{Nb} / \mathrm{Cu}}}
$$

Following standard procedures of inverse problem solution, we can now discretize the integral equation (2) in order to reduce the original problem to the solution of a linear set of algebraic equations:

$$
\begin{aligned}
& \overline{R_{S}\left(T_{o}, E_{\mathrm{acc} 1}\right)}=\Gamma / Q_{1}=\sum_{i=1}^{N} R_{S}\left(T_{o}, E_{\mathrm{acc} 1}, R_{\mathrm{Nb} / \mathrm{Cu} i}\right) f_{i} \Delta R_{\mathrm{Nb} / \mathrm{Cu} i} \\
& \overline{R_{S}\left(T_{o}, E_{\mathrm{acc} j}\right)}=\Gamma / Q_{j}=\sum_{i=1}^{N} R_{s}\left(T_{o}, E_{\mathrm{acc} j}, R_{\mathrm{Nb} / \mathrm{Cu} i}\right) f_{i} \Delta R_{\mathrm{Nb} / \mathrm{Cu} i} \\
& \overline{R_{s}\left(T_{o}, E_{\mathrm{acc} N}\right)}=\Gamma / Q_{N}=\sum_{i=1}^{N} R_{s}\left(T_{o}, E_{\mathrm{acc} N}, R_{\mathrm{Nb} / \mathrm{Cu} i}\right) f_{i} \Delta R_{\mathrm{Nb} / \mathrm{Cu} i}
\end{aligned}
$$


with $E_{\text {acc } j}, Q_{j}$ being the $N$ measured experimental data points, $f_{i}$ and $R_{\mathrm{Nb} / \mathrm{Cu} i}$ the discretized variables of Eq. (2), and with the normalization condition which now reads: $\sum_{i=1}^{N} f_{i} \Delta R_{\mathrm{Nb} / \mathrm{Cu} i}=1$.

The above system of equations is equivalent to saying that the measured increase from $R_{s 0}$ of the average surface resistance at a given field $E_{\text {acci }}$ is due to the fraction of surface that has transitioned to normal state up to that field level. This is made explicit by using for $R_{s}=R_{s}\left(T_{o}, E_{\mathrm{acc}}, R_{\mathrm{Nb} / \mathrm{Cu}}\right)$ the functional dependence (5) and by setting $R_{\mathrm{Nb} / \mathrm{Cu} i}=K / E_{\mathrm{acc}(N+1-i)}^{2}$ from Eq. (6). (Note that with this choice the index of $R_{\mathrm{Nb} / \mathrm{Cu} i}$ decreases when the index of $E_{\operatorname{acc}(N+1-i)}$ increases). We then get:

$$
\begin{aligned}
& \overline{R_{s}\left(T_{o}, E_{\mathrm{acc} 1}\right)}=\sum_{i=1}^{N} R_{s o} f_{i} \Delta R_{\mathrm{Nb} / \mathrm{Cu} i} \\
& \overline{R_{S}\left(T_{o}, E_{\mathrm{acc} j}\right)}=\sum_{i=1}^{N-j+1} R_{s o} f_{i} \Delta R_{\mathrm{Nb} / \mathrm{Cu} i}+\sum_{i=N-j+2}^{N} R_{n} f_{i} \Delta R_{\mathrm{Nb} / \mathrm{Cu} i} \\
& \overline{R_{S}\left(T_{o}, E_{\mathrm{acc} N}\right)}=R_{s o} f_{1} \Delta R_{\mathrm{Nb} / \mathrm{Cu} 1}+\sum_{i=2}^{N} R_{n} f_{i} \Delta R_{\mathrm{Nb} / \mathrm{Cu} i}
\end{aligned}
$$

where it is assumed $E_{\mathrm{acc} 1}<E_{\mathrm{acc} j}<E_{\mathrm{acc} N}$ and $R_{\mathrm{Nb} / \mathrm{Cu} N}>$ $R_{\mathrm{Nb} / \mathrm{Cu} j}>R_{\mathrm{Nb} / \mathrm{Cu} 1}$.

Subtracting from each equation the previous one (starting from the last one) we get:

$$
\begin{aligned}
f_{2} \Delta R_{\mathrm{Nb} / \mathrm{Cu} 2}= & \frac{\overline{R_{s}\left(T_{o}, E_{\mathrm{acc} N}\right)}-\overline{R_{s}\left(T_{o}, E_{\mathrm{acc}(N-1)}\right)}}{R_{n}-R_{s o}} \\
f_{N} \Delta R_{\mathrm{Nb} / \mathrm{Cu} N}= & \frac{\overline{R_{s}\left(T_{o}, E_{\mathrm{acc} 2}\right)}-\overline{R_{S}\left(T_{o}, E_{\mathrm{acc} 1}\right)}}{R_{n}-R_{s o}}
\end{aligned}
$$

The value of $f_{1} \Delta R_{\mathrm{Nb} / \mathrm{Cu} 1}$ can be obtained from the normalization condition $\sum_{i=1}^{N} f_{i} \Delta R_{\mathrm{Nb} / \mathrm{Cu} i}=1$.

In this way the distribution function $f\left(R_{\mathrm{Nb} / \mathrm{Cu}}\right)$ is extracted in the form of a discrete distribution with the number of points equal to $\mathrm{N}-1$. The fractional area $A$ presenting a degraded thermal impedance is thus $A=\sum_{i=2}^{N} f_{i} \Delta R_{\mathrm{Nb} / \mathrm{Cu} i}$.

In order to assess the validity of this streamlined inversion procedure based on the simplification allowed by Eq. (5), we have checked it against the full inversion process described in the previous paper [7] using as a benchmark the rf data of cavity QS7.2. The difference for the fractional area $A$ calculated with the two methods is less than $5 \%$, well within the typical experimental uncertainty of RF measurements.

\section{RESULTS AND DISCUSSION}

The HIE-ISOLDE QWR cavities have been characterized by the usual $Q\left(E_{\text {acc }}\right)$ measurements (Fig. 1), from which the values of average surface resistance were extracted using the geometrical factor of $30.8 \mathrm{Ohm}$, without taking into account the spatial variations of the surface fields. The surface resistance data have been selected among those displaying the lowest thermal gradient across the cavity upon cooldown [6] in order to minimize unwanted extrinsic effects. The surface resistance of the different cavities has been fitted up to $3 \mathrm{MV} / \mathrm{m}$ following standard practice [9] as $R_{s}(E)=R_{s}^{0}+R_{s}^{1} E_{\text {acc }}$ in order to extract the relevant coefficients for further comparison.

Calculation of the distribution function $f\left(R_{\mathrm{Nb} / \mathrm{Cu}}\right)$ following the inversion procedure described in the previous section has been performed using only data points in the interval $(0,5) \mathrm{MV} / \mathrm{m}$, for better systematic comparison among the different cavities, and also to avoid any effect of electron field emission, which at the highest fields might have influenced the rf measurements. Although the inversion procedure could be performed on the raw rf data, these have been smoothened to have monotonic Rs(E) data sets. For each data set, the zero-field surface resistance $R_{s 0}$ is assumed equal to the value of $R_{s}^{0}$ mentioned above, which is consistent with the procedure since the model is dealing with local BCS surface resistance increases relative to the temperature of $4.5 \mathrm{~K}$. The average surface resistance is indeed dominated by the residual component, the BCS

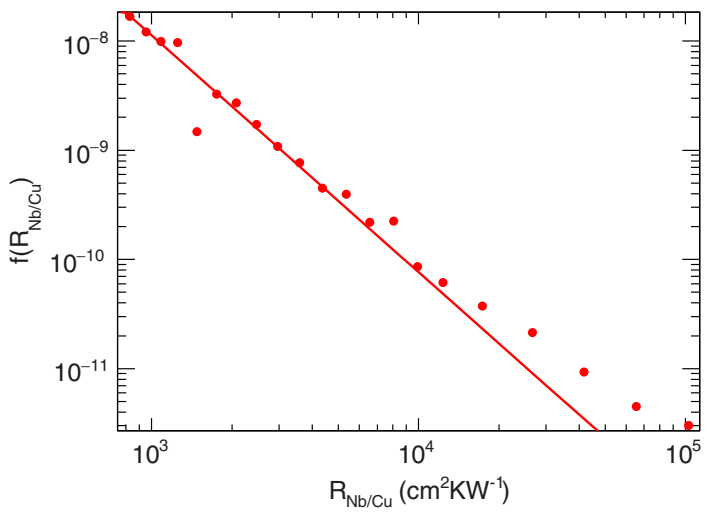

FIG. 6. Typical result of the fitting procedure of the thermal model (cavity QP1.4 in this example). 


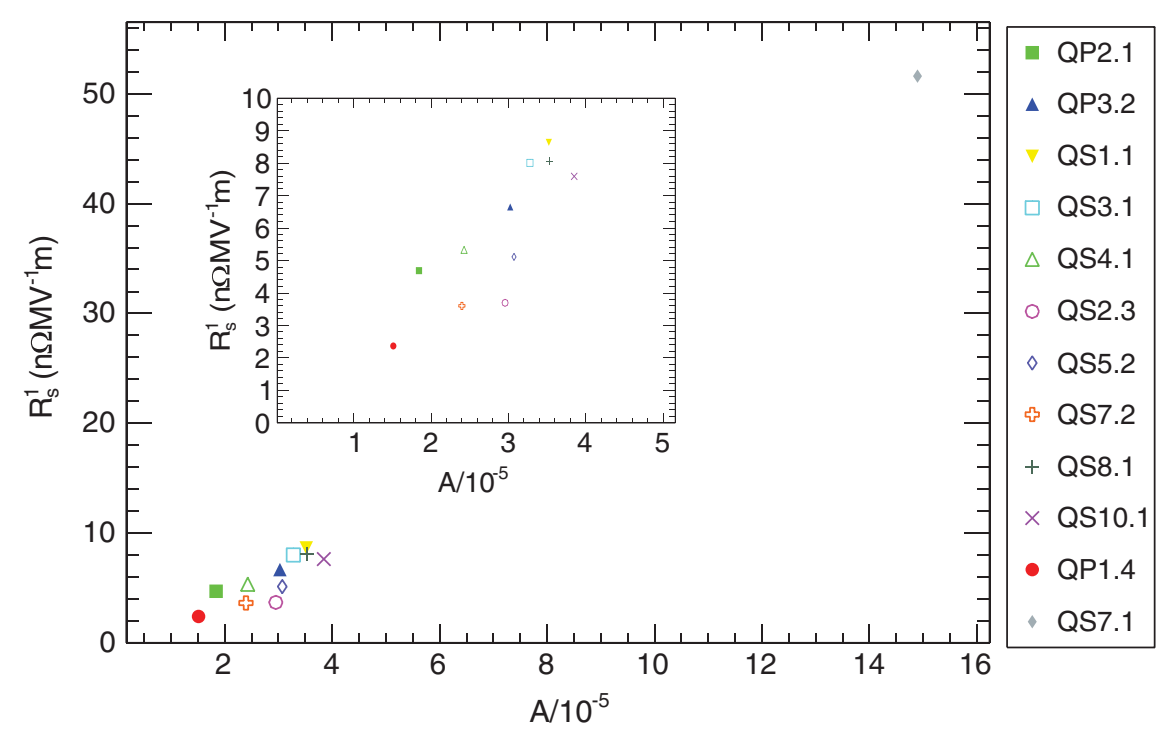

FIG. 7. Plot of $R_{s}^{1}$ as a function of $A$. The insert allows an expanded view of all cavities except QS7.1.

component of our thin films at $4.5 \mathrm{~K}$ being only about $10 \mathrm{nOhm}$. This assumption also means implicitly that $R_{s}^{0}$ is related only to the intrinsic film properties, while $R_{s}^{1}$ is related to thermal boundary properties described by the model.

Plots of the distribution function $f\left(R_{\mathrm{Nb} / \mathrm{Cu}}\right)$ as a function of the interface thermal impedance $R_{\mathrm{Nb} / \mathrm{Cu}}$ display in general a linear behavior when represented in a log-log scale, as illustrated in Fig. 6, with one notable exception discussed below (cavity QS7.1). This allows fitting the data with a power law of the form $f(R)=\alpha R^{\beta}$.

Correlation plots have been established among the quantities extracted from the data inversion process, namely $(A, \alpha, \beta)$ and the measured values of $R_{s}^{0}$ and $R_{s}^{1}$. A significant correlation $(r=0.994)$ has been found only between the fractional area $A$ and the coefficient $R_{s}^{1}$ which represents the "slope" of the $R_{S}(E)$ curves. This is illustrated in Fig. 7. Such a correlation, together with the absence of correlation between $R_{s}^{1}$ and both $\alpha$ and $\beta$, suggests that the difference among all cavities is only in the total amount of defects that generate a locally degraded thermal impedance, but not in their nature.

Past investigations suggest that pinholes from manufacturing or surface preparation, which may also release chemicals, as well as dust particles, could be among the possible sources of such defects $[10,11]$. The latter phenomenon is particularly noteworthy considering that the two cavities showing the lowest values for $A$ are indeed the two prototype cavities of the "QP" series, which were free of manufacturing defects. It should also be mentioned that, although great care is taken when preparing the substrates in a clean room, defects created by dust particles at the $\mathrm{Nb} / \mathrm{Cu}$ interface might still be an intrinsic limitation of all coatings. In particular, we could note that the density of dust particle sizes deposited onto surfaces in clean rooms [12] also follows a power law distribution.

As mentioned above, a notable exception to the standard behavior is the cavity coating labeled QS7.1 performed using a larger bias voltage $(-120 \mathrm{~V})$, and resulting in lower rf performance compared to all other cavities. The cavity
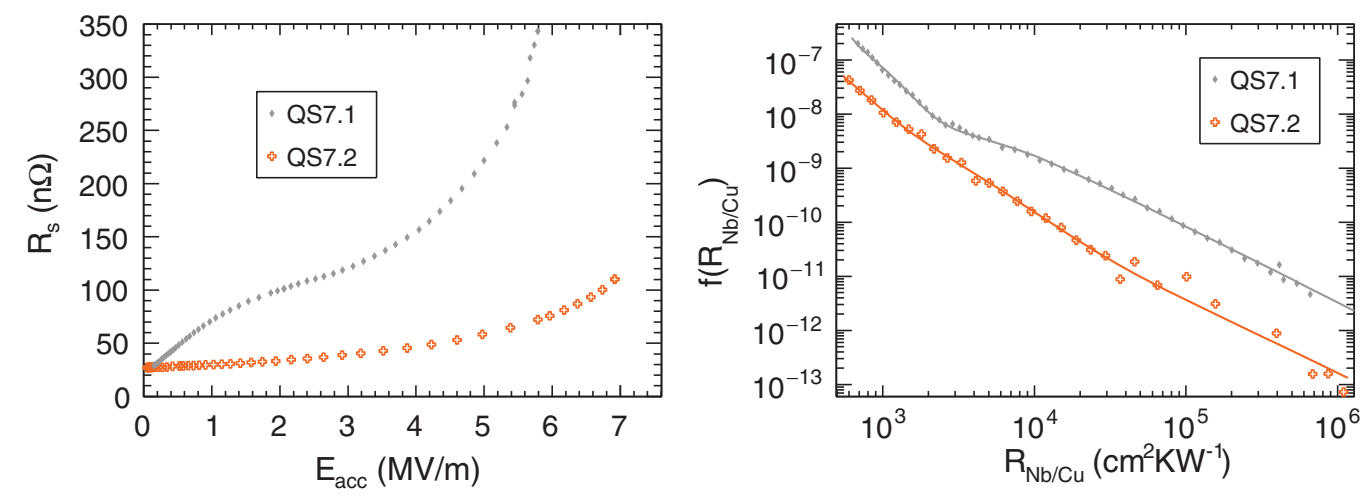

FIG. 8. $\quad R_{s}\left(E_{\mathrm{acc}}\right)$ curves for QS7.1 and QS7.2 (left) and their distribution function $f\left(R_{\mathrm{Nb} / \mathrm{Cu}}\right)$ as a function of the interface thermal impedance $R_{\mathrm{Nb} / \mathrm{Cu}}$ (right). The lines are only intended as a guide for the eye. 
was also coated a second time (labeled QS7.2) according to the standard procedure with a bias of $-80 \mathrm{~V}$, after stripping of the first $\mathrm{Nb}$ film. By making use of the same substrate, one may assume that any difference in performance should depend essentially on the film properties, and not on features of the substrate itself. The $\operatorname{Rs}\left(E_{\mathrm{acc}}\right)$ data of the two coatings are compared in Fig. 8 together with plots of the distribution function $f\left(R_{\mathrm{Nb} / \mathrm{Cu}}\right)$. Cavity QS7.1 displays a two slopes behavior, which might be linked to the delamination identified in the cross sectional analyses mentioned in Sec. II (high values of thermal impedance could in fact be expected for extended delaminations of the film).

\section{CONCLUSION}

The increase of surface resistance with accelerating field of $\mathrm{Nb} / \mathrm{Cu}$ cavities can be successfully described in terms of a recent model, which assumes the existence of regions of locally enhanced thermal impedance at the $\mathrm{Nb} / \mathrm{Cu}$ interface [7].

This model has been reformulated in this paper for a greater flexibility in the data-analysis procedure, and successfully applied to describe the rf performance data of the first production batch of the HIE-ISOLDE cavities. A correlation has been found between the fractional area $A$ presenting a degraded thermal impedance and the "slope" of the surface resistance with increasing field described by the quantity $R_{s}^{1}$. Moreover, some evidence is presented that cavities showing defects of different nature (surface defects, localized film delaminations) display a distinctive behavior in terms of the characteristic distribution function $f\left(R_{\mathrm{Nb} / \mathrm{Cu}}\right)$ defined by the model.

These are important first elements upholding the validity of the model, however more data and possibly ad-hoc experiments would be needed for a complete and direct verification.

\section{ACKNOWLEDGMENTS}

Many colleagues have contributed to the HIE-ISOLDE project; the authors would like to acknowledge in particular the contribution of Pei Zhang for RF tests and of Barbora Bártová and the Interdisciplinary Centre for Electron Microscopy (CIME) of Ecole Polytechnique Fédérale de Lausanne (EPFL) for microscopic analyses. V.P. and
R. V. have been supported by the INFN V Group Experiment ISIDE.

[1] M. Pasini et al., A SC upgrade for the REX-ISOLDE accelerator at CERN, in Proceedings of the LINAC08 Conference, Victoria, 2008 (JACoW, Geneva, 2008), p. 124.

[2] C. Benvenuti, N. Circelli, and M. Hauer, Niobium films for superconducting accelerating cavities, Appl. Phys. Lett. 45, 583 (1984).

[3] W. Venturini Delsolaro et al., Nb sputtered quarter wave resonators for the HIE-ISOLDE, in Proceedings of the SRF2013 Conference, Paris, 2013 (JACoW, Geneva, 2013), p. 767.

[4] N. Jecklin et al., Niobium coatings for the HIE-ISOLDE QWR superconducting accelerating cavities, in Proceedings of the SRF2013 Conference, Paris, 2013 (JACoW, Geneva, 2013), p. 611.

[5] A. Sublet et al., Nb coated HIE-ISOLDE QWR superconducting accelerating cavities: From process development to series production, in Proceedings of the IPAC2014 Conference, Dresden, 2014 (JACoW, Geneva, 2014), p. 2571.

[6] P. Zhang et al., The influence of cooldown conditions at transition temperature on the quality factor of niobium sputtered quarter-wave resonators for HIE-ISOLDE, in Proceedings of the SRF 2015 Conference, Whistler, 2015 (JACoW, Geneva, 2015), p. 765.

[7] V. Palmieri and R. Vaglio, Thermal contact resistance at the $\mathrm{Nb} / \mathrm{Cu}$ interface as a limiting factor for sputtered thin film RF superconducting cavities, Supercond. Sci. Technol. 29, 015004 (2016).

[8] L. Alberty et al., The copper substrate developments for the HIE-ISOLDE high-beta quarter wave resonator, in Proceedings of the SRF2013 Conference (Paris, 2013), p. 596.

[9] C. Benvenuti, S. Calatroni, I. E. Campisi, P. Darriulat, M. A. Peck, R. Russo, and A.-M. Valente, Study of the surface resistance of superconducting niobium films at $1.5 \mathrm{GHz}$, Physica (Amsterdam) 316C, 153 (1999).

[10] S. Amorosi, C. Benvenuti, P. Chiggiato, and M. Malabaila, Experimental evaluation of niobium film pinholes, Vacuum 60, 275 (2001).

[11] C. Benvenuti, S. Calatroni, P. Darriulat, M. A. Peck, A.-M. Valente, and C. A. Van't Hof, Study of the residual surface resistance of niobium films at $1.5 \mathrm{GHz}$, Physica (Amsterdam) 351C, 421 (2001).

[12] ISO standard 14664. 